\title{
Efeito do eCG ou benzoato de estradiol associado ao norgestomet na taxa de concepção de vacas de corte submetidas à IATF no pós-parto
}

Luis Augusto Ferreira

ROSSA $^{1}$

Claudia Maria BERTAN²

Alexandre Barreto de

ALMEIDA $^{1}$

Pércio dos Santos GASPAR ${ }^{1}$

Paulo Henrique MAZZA ${ }^{3}$

Mario BINELLI ${ }^{1}$

Pietro Sampaio BARUSELLI ${ }^{1}$

Ed Hoffmann MADUREIRA ${ }^{1}$

\section{Correspondência para:}

Rua Duque de Caxias Norte, 225, Departamento de Reprodução Animal Centro de Biotecnologia em Reprodução Animal - FMVZ-USP, Pirassununga/SP 13630-000, (19) 35654019, Fax (19)

35654060; madureh@usp.br

Recebido para publicação: 19/05/2005 Aprovado para publicação: 30/04/2009

\author{
1 - Departamento de Reprodução Animal da Faculdade de Medicina Veterinária e \\ Zootecnia da Universidade de São Paulo, Pirassununga-SP \\ 2 - Faculdade de Zootecnia da Universidade Estadual Paulista, Dracena-SP \\ 3 - Departamento de Nutrição e Produção Animal da Faculdade de Medicina \\ Veterinária e Zootecnia da Universidade de São Paulo, Pirassununga-SP
}

\section{Resumo}

Este estudo objetivou comparar as taxas de concepção, em vacas de corte no período pós-parto (PPP), tratadas com Gonadotrofina Coriônica Eqüina (eCG) ou Benzoato de Estradiol (BE), após o uso de Norgestomet submetidas à inseminação artificial em tempo fixo (IATF). A hipótese é que a administração de eCG ou BE aumenta as taxas de concepção. Vacas Nelore $(\mathrm{n}=138)$ e Brangus ( $\mathrm{n}=63)$, lactantes, entre 26 e 118 dias do PPP, foram divididas em três grupos homogêneos e dentro de cada grupo subdivididas em dois blocos, um com um PPP < 45 dias (PPP1; $\mathrm{n}=107$ ) e outro > 45 dias (PPP2; $\mathrm{n}=94)$. Todas as fêmeas receberam um implante auricular contendo $3 \mathrm{mg}$ de Norgestomet $\left(\right.$ Crestar $^{\mathbb{B}}$ ), seguido pela administração de $5 \mathrm{mg}$ de Valerato de Estradiol. Os implantes auriculares foram mantidos durante 10 dias. Na retirada dos implantes, as fêmeas receberam $1 \mathrm{~mL}$ de solução fisiológica ( $\mathrm{n}=68$; Grupo Controle) ou 500UI de eCG (Folligon $^{\circledR}$; n=67; Grupo eCG) ou $1 \mathrm{mg}$ de Benzoato de Estradiol (Index; n=66; Grupo BE) 24 horas após a retirada do implante. A IATF foi realizada 54 horas após a retirada do implante. O diagnóstico de gestação foi realizado por ultra-sonografia 30 dias após a IATF. Houve interação entre os tratamentos e o PPP. No PPP1, a taxa de concepção foi maior no grupo eCG comparado ao BE (47,22\% vs. 15,38\%; P<0,01). No PPP2, as taxas de concepção dos grupos eCG e BE foram maiores que no grupo controle $(41,93 \%, 44,44 \%$ vs. $22,22 \% ; \mathrm{P}<0,01)$. Conclui-se que vacas, com até 45 dias do PPP, o eCG associado ao Norgestomet aumenta as taxas de concepção.

\section{Introdução}

Em rebanhos bovinos a eficiência reprodutiva das fêmeas está diretamente relacionada à produção de bezerros. Para um rebanho comercial obter máxima produtividade, o ideal seria cada vaca produzir um bezerro por ano. Para o estabelecimento do intervalo entre partos de 12 meses, torna-se fundamental o emprego da inseminação artificial em tempo fixo (IATF), procedimento que proporciona uma alta taxa de serviço além de tornar desnecessária a detecção de estros. ${ }^{1}$
Palavras-chave: Norgestomet. eCG. Benzoato de estradiol. Pós-parto. Vacas.
Considerando um período de gestação médio de 290 dias em fêmeas zebuínas, para a produção de um bezerro/ ano, o intervalo entre o parto e a próxima concepção deve ser no máximo de 75 dias. ${ }^{2}$ Entretanto, no referido período, a condição de anestro acomete a maioria das fêmeas, determinando uma redução nas taxas de prenhez e na eficiência reprodutiva dos rebanhos. ${ }^{3}$ No sistema extensivo de criação de gado de corte nos Estados Unidos, assim como no Brasil, aproximadamente $50 \%$ das vacas apresentam anestro no início da estação de monta. ${ }^{4,5} \mathrm{Em}$ vacas de corte, a 
duração do anestro pós-parto é afetada por vários fatores, dentre os principais: e número de parições ${ }^{3}$, problemas nutricionais que resultam em vacas com baixo escore de condição corporal ${ }^{6}$; amamentação ${ }^{7} \mathrm{e}$ incidência de ciclos curtos ${ }^{8}$ e número de parições ${ }^{3}$.

Os mecanismos determinantes do anestro pós-parto envolvem uma complexa relação entre hipotálamo, hipófise, ovários e útero." A exposição prolongada a altas concentrações de estrógeno $\left(\mathrm{E}_{2}\right)$ e progesterona $\left(\mathrm{P}_{4}\right)$ no final da gestação promovem um "feedback" negativo no hipotálamo, evento fisiológico que determina uma redução nos estoques hipofisários do hormônio luteinizante ( $\mathrm{LH})$ e hormônio folículo estimulante (FSH). ${ }^{9}, 10$ Após o parto, os estoques de FSH são restabelecidos rapidamente, condição que permite o recrutamento e seleção do folículo dominante ${ }^{7,11}$; entretanto, o mesmo não ocorre com o LH. A ovulação do folículo dominante torna-se possível apenas quando houver o restabelecimento da freqüência adequada dos pulsos de LH. ${ }^{10} \mathrm{O}$ padrão pulsátil de liberação de LH pós-parto se caracteriza por baixa freqüência, com a ocorrência de menos de um pulso de $\mathrm{LH}$ a cada 4 horas. ${ }^{12}$ Tal freqüência aumenta durante o período imediatamente anterior à ovulação até aproximadamente um pulso de LH a cada 1 a 2 horas. ${ }^{13}$ Normalmente, o anestro é determinado pela baixa freqüência dos pulsos de $\mathrm{LH}$, condição que limita o crescimento folicular e a ovulação. ${ }^{14,15}$

Alguns autores reportaram que 30 dias após o parto ocorre um aumento na liberação de GnRH e consequentemente nos pulsos de $\mathrm{LH} .{ }^{16} \mathrm{O}$ aumento na pulsatilidade de LH é necessário para que os folículos adquiram dominância e capacidade de secretarem grandes quantidades de $\mathrm{E}_{2}$, evento fisiológico determinante do "feedback" positivo no hipotálamo. ", 17 Vacas de corte, com boa condição corporal, possuem um intervalo de 27 a 37 dias entre o parto e a primeira ovulação. Tal intervalo é prolongado, para 60 a 120 dias, em vacas com condição corporal ruim. ${ }^{18}$ Wettmann et $a .^{7}$ relataram que vacas com comprometimento nutricional parecem ser mais sensíveis aos efeitos do "feedback" negativo do $\mathrm{E}_{2}$.

Reportou-se que a utilização de $\mathrm{P}_{4}$ exógena ou progestágenos antecipou a retomada da ciclicidade em vacas no período pós-parto. O tratamento com Norgestomet aumentou a freqüência dos pulsos de $\mathrm{LH}^{19}$, as concentrações de $E_{2}$ no soro sangüíneo ${ }^{16,}$ ${ }^{20}$ e no fluído folicular ${ }^{19}$. Além disso, tais hormônios promoveram um "priming" no útero que previniram a ocorrência de ciclos interovulatórios de curta duração. ${ }^{10}$ Contudo, quando vacas em anestro pós-parto com condição nutricional ruim, foram tratadas apenas com $\mathrm{P}_{4}$ ou progestágenos, reduzidas taxas de prenhez foram registradas. ${ }^{21} \mathrm{O}$ tratamento com Norgestomet durante nove dias em vacas em anestro, entre 3 e 78 dias pós-parto, resultou em uma taxa de prenhez de apenas $17 \%{ }^{22}$

Foi demonstrado em vários estudos que a administração de 300 a 400UI de Gonadotrofina Coriônica Eqüina (eCG), associada a progestágenos, em vacas de corte com até 60 dias pós-parto, favorece as taxas de concepção, quando as fêmeas são submetidas à IATF. Relatou-se que os folículos dominantes no período pós-parto são menores que os ovulatórios que precedem os ciclos curtos ou normais, possivelmente porque os folículos dominantes não ovulatórios no pós-parto sofrem atresia antes de atingirem o tamanho requerido para a ovulação. ${ }^{23}$ Reportou-se que o eCG é capaz de se ligar tanto aos receptores de LH quanto de FSH. ${ }^{24}$ Desta forma, o eCG é capaz de incrementar o crescimento final do folículo dominante e estimular a síntese de $\mathrm{E}_{2}$. Alguns autores verificaram um incremento no grau de sincronização com redução significativa da variação no tempo para o pico préovulatório de LH e ovulação, quando administraram 400UI de eCG na retirada do implante de Norgestomet. ${ }^{25}$ A associação do eCG é recomendada em vacas com condição corporal menor que 5 (escala de 1 a 9), em intervalo pós-parto menor do 
que 50 dias e em fêmeas na condição de anestro pós-parto. ${ }^{8}$

Em fêmeas tratadas com progestágenos, a administração de benzoato de estradiol (BE) promove uma sincronização no pico de LH e nas ovulações, entretanto, tal função foi dependente do estágio do folículo dominante no momento do tratamento. ${ }^{26}$ Verificou-se em alguns estudos que a sincronização da ovulação com BE, pode ser útil em animais cíclicos e com boa condição corporal. ${ }^{27} \mathrm{De}$ fato, alguns autores sugerem que a aplicação de $\mathrm{BE}$ não pode substituir a aplicação de eCG no tratamento de sincronização com Norgestomet em vacas Bos taurus indicus com cria ao pé, em rebanhos com alta percentagem de fêmeas em anestro. ${ }^{25}$

Assim, este estudo objetivou comparar as taxas de concepção em vacas de corte no período pós-parto tratadas com progestágenos, associado ou não ao uso de eCG ou BE. A hipótese do presente estudo é que a utilização do eCG ou BE, associado ao Norgestomet, promove um incremento nas taxas de concepção em fêmeas submetidas à IATF.

\section{Material e Método}

O experimento foi desenvolvido em duas fazendas. A primeira sediada no município de Rancharia, estado de São Paulo, onde foram utilizadas 63 vacas da raça Brangus (Bos taurus taurus $\mathrm{x}$ Bos taurus indicus), pluríparas, amamentando, com um período pós-parto compreendido entre 36 e 118 dias, média de 77 dias. As vacas foram mantidas em Brachiaria brizanta var marandu e receberam suplementação mineral ad libitum. A segunda fazenda, localizada no município de Martinópolis, estado de São Paulo, o experimento procedeu-se com 138 vacas da raça Nelore (Bos taurus indicus), pluríparas, amamentando, com um período pós-parto entre 26 e 63 dias, em média 44 dias. As fêmeas permaneceram em piquetes formados por Brachiaria decumbens e receberam suplementação mineral ad libitum. Do total de 201 vacas utilizadas neste estudo,
107 fêmeas apresentavam período pós-parto $<45$ dias (PPP1) e 94 vacas $>45$ dias (PPP2). Considerando a classificação de escore de condição corporal (ECC) de 1 a $9^{28}$, o ECC médio foi de 4,11 + 0,71 para PPP1 e 4,14 + 0,67 para PPP2. Considerando a condição ovariana das vacas, segundo a classificação de 1 a $3^{8}$, a mesma foi de $1(n=13), 2(n=45)$ e $3(n=49)$ para PPP1 e de $1(n=25), 2(n=47)$ e $3(n=22)$ para PPP2. Em tal classificação, 1 refere-se às fêmeas ciclando com folículos $>10 \mathrm{~mm}$ com ou sem a presença de corpo lúteo; 2 às fêmeas que possuem folículos que atingem a fase de dominância $(>8,5 \mathrm{~mm})$ mas não são capazes de ovular e 3 as fêmeas com folículos pequenos, lisos e duros condição em que os folículos não chegam até a fase de divergência. ${ }^{8}$

O delineamento utilizado foi o de blocos casualizados generalizados. As fêmeas foram divididas equitativamente, segundo os escores de condição corporal e condição ovariana em três grupos homogêneos (Grupo Controle, Grupo eCG e Grupo BE). Dentro de cada grupo as vacas foram subdivididas em dois blocos, um com PPP $<45$ dias (PPP1) e outro $>45$ dias (PPP2). Todas as fêmeas receberam um implante de silicone contendo $3 \mathrm{mg}$ de Norgestomet (Kit Crestar ${ }^{\circledR}$ - Intervet) na face auricular externa, seguido pela administração de $5 \mathrm{mg}$ de Valerato de Estradiol (Kit Crestar ${ }^{\circledR}$ Intervet), via intramuscular. $\mathrm{O}$ dia da implantação do implantes foi considerado o D0. Os implantes auriculares foram mantidos durante 10 dias. Na retirada dos implantes (D10), as fêmeas receberam $1 \mathrm{~mL}$ de solução fisiológica ( $\mathrm{n}=68$; Grupo Controle) via subcutânea ou 500UI de eCG (Folligon ${ }^{\circledR}$ - Intervet; $n=76$; Grupo eCG) via subcutânea ou $1 \mathrm{mg}$ de Benzoato de Estradiol (Index Farmacêutica; $n=66$; Grupo BE) via intramuscular, 24 horas após a retirada do implante. A IATF foi realizada 54 horas após a retirada do implante. O diagnóstico de gestação foi realizado por ultra-sonografia (Aloka 500, Japão; Probe $7,5 \mathrm{MHz}) 30$ dias após a IATF. No modelo estatístico considerou-se como variável dependente a taxa de concepção (TC), 
determinada pelo número de fêmeas prenhes em relação ao total de vacas submetidas à IATF. Foram variáveis independentes: Fazenda (1 ou 2), tratamento (Grupo Controle; Grupo eCG e Grupo BE), período pós-parto (PPP1 e PPP2) e as possíveis interações (fazenda $\mathrm{x}$ tratamento; fazenda x PPP; tratamento x PPP; fazenda $\mathrm{x}$ tratamento $\mathrm{x}$ PPP). Os dados foram analisados por análise de variância empregando-se o Programa Computacional SAS ${ }^{29}$. Na presença de efeito do tratamento, a comparação entre as médias foi realizada por contrastes ortogonais. Para a interação tratamento x PPP, as comparações foram realizadas por contrastes dentro de cada período pós-parto. Admitiu-se um nível de significância de $5 \%$ para os testes realizados.

\section{Resultados e Discussão}

Os resultados obtidos neste estudo estão apresentados na tabela 1. No PPP1, quando o grupo eCG foi associado ao $\mathrm{BE}$ e ambos comparados ao grupo controle, verificou-se uma tendência dos grupos eCG
/BE promoverem maior TC comparado ao grupo controle $(47,22 \% / 15.38 \%$ vs. $12,50 \% ; \mathrm{P}=0,0597)$. No PPP2, os grupos eCG/BE resultaram em maiores TC comparados ao grupo controle $(41,93 \%$ / $44,44 \%$ vs. $22.22 \%$; P <0,01). No PPP1, quando o grupo eCG foi comparado ao $\mathrm{BE}$, verificou-se que a TC do grupo eCG foi maior que $\mathrm{BE}(47,22 \%$ vs. $15,38 \%$, $\mathrm{P}<0,01)$, entretanto, o mesmo não foi observado para o PPP2 (41,93\% vs. 44,44\%; $\mathrm{P}>0,05)$.

Para os animais tratados com eCG verificou-se uma taxa de concepção de $47,22 \%$ no PPP1 e $41,93 \%$ no PPP 2 . Em outro estudo verificou-se em vacas de corte, tratadas com eCG associado ao Norgestomet no período pós-parto de 90 dias, uma taxa de concepção de $48,6 \%{ }^{30} \mathrm{O}$ mesmo não foi observado quando associaram 500UI de eCG ao Norgestomet, em vacas de corte em anestro, no período compreendido entre 70 e 90 dias pós-parto, onde a taxa de concepção foi de $14 \%{ }^{31}$ Outros autores verificaram que $750 \mathrm{UI}$ de eCG associado ao progestágeno, em vacas

Tabela 1 - Taxas de concepção em vacas Brangus (Bos taurus taurus x Bos taurus indicus) e Nelore (Bos taurus indicus), pluríparas, lactantes, no período pós parto, tratadas com implante auricular contendo 3mg de Norgestomet associado a administração de $5 \mathrm{mg}$ de Valerato de Estradiol via IM, no momento da colocação do implante; seguida pela remoção dos implantes 10 dias após, momento em que as fêmeas receberam $1 \mathrm{~mL}$ de solução fisiológica via SC (Grupo Controle; $\mathrm{n}=68$ ) ou 500UI de eCG via SC (Grupo eCG; $n=67$ ) ou $1 \mathrm{mg}$ de Benzoato de Estradiol via IM 24 horas após a retirada do implante (Grupo BE; $\mathrm{n}=66$ ), todas submetidas à IATF 54 horas após a retirada do implante - Rancharia/SP e Martinópolis/ SP - 2000

\begin{tabular}{|c|c|c|}
\hline Período Pós Parto & Tratamento & Taxa de Concepção (\%) \\
\hline & Grupo Controle $(\mathrm{n}=32)$ & $12,50(4 / 32)$ \\
\hline \multirow[t]{3}{*}{ PPP $1(n=107)$} & Grupo eCG $(n=36)$ & $47,22(17 / 36)$ \\
\hline & Grupo BE $(n=39)$ & $15,38(6 / 39)$ \\
\hline & Grupo Controle $(\mathrm{n}=36)$ & $22,22(8 / 36)$ \\
\hline \multirow[t]{2}{*}{ PPP $2(n=94)$} & Grupo eCG $(\mathrm{n}=31)$ & $41,93(13 / 31)$ \\
\hline & Grupo $B E(n=27)$ & $44,44(12 / 27)$ \\
\hline \multicolumn{2}{|c|}{ Contrastes Ortogonais: Tratamento $\mathrm{x}$ Período Pós Parto } & $P$ \\
\hline \multicolumn{2}{|c|}{ PPP 1 - Grupo Controle x eCG / BE } & 0,0597 \\
\hline \multicolumn{2}{|c|}{ PPP 1 - Grupo eCG x BE } & 0,0016 \\
\hline \multicolumn{2}{|c|}{ PPP 2 - Grupo Controle x eCG / BE } & 0,0179 \\
\hline \multicolumn{2}{|c|}{ PPP 2 - Grupo eCG x BE } & 0,7759 \\
\hline
\end{tabular}

* PPP1 $=$ Período Pós Parto $\leq 45$ dias) $/$ PPP2 $=$ Período Pós Parto $>45$ dias 
com 89 dias pós-parto, determinou uma taxa de concepção $73 \%{ }^{32}$

Verificou-se que folículos no $4^{\circ}$ dia de desenvolvimento apresentam um significativo aumento no número de receptores para LH nas células da teca e granulosa. ${ }^{33}$ Também se verificou um aumento significativo no número de receptores de FSH no mesmo período. ${ }^{34}$ Segundo Inkeep et al. ${ }^{35}$, o Norgestomet promove em folículos dominantes um aumento no número de receptores de $\mathrm{LH}$ nas células da teca e granulosa. No presente experimento, empregou-se o implante de Norgestomet durante 10 dias acompanhado da administração de Valerato de Estradiol no dia da inserção do implante. Considerando que tal associação de fármacos favorece a emergência de uma nova onda folicular 6,3 dias após ${ }^{36}$, era esperado que no momento da aplicação do eCG o folículo dominante estivesse entre o $4^{\circ}$ e $5^{\circ}$ dia pós emergência. Considerando as observações realizadas por alguns autores ${ }^{35}$, em tal momento, haveria grande concentração de receptores para o LH no folículo dominante, condição que o torna altamente responsivo ao eCG, assim, tal fármaco estimularia o desenvolvimento final do folículo dominante e possivelmente, desta maneira, auxiliaria a sincronização da ovulação.

Alguns trabalhos têm demonstrado que o uso de eCG incrementa as taxas de concepção em vacas no período pós-parto com baixo escore corporal ${ }^{32,37,38}$; entretanto, tal incremento não foi observado em vacas ciclando e com boa condição corporal ${ }^{39,40}$. No presente estudo, a condição corporal das fêmeas era bastante homogênea, assim não pode ser avaliado o efeito do eCG em vacas com diferentes escores de condição corporal.

No presente estudo, observou-se uma interação entre o tratamento e o período pós-parto. No PPP1, a administração de eCG foi capaz de elevar a TC quando comparado ao $\mathrm{BE}(47,22 \%$ vs. $15,38 \%, \mathrm{P}$ $<0,01)$. De fato, alguns autores relataram que os folículos tornam-se responsivos as gonadotrofinas exógenas em um intervalo pós-parto inferior a 30 dias. ${ }^{3,41}$ Segundo Crowe et al. ${ }^{42}$, o eCG ao suprir a falta de $\mathrm{LH}$, proporciona ao folículo a capacidade de se desenvolver até a fase pré-ovulatória, considerando que tal evento é dependente de $\mathrm{LH}^{43}$. Verificou-se que a ativação dos receptores de $\mathrm{LH}$ e FSH no folículo promove um aumento na concentração de adenosina monofosfato cíclico (AMPc) intrafolicular, evento que promoveria a ativação de enzimas intracelulares que contribuiriam para incrementar produção de $\mathrm{E}_{2}$ pelo folículo. ${ }^{44}$ Observou-se que o aumento gradativo de $\mathrm{E}_{2}$ plasmático ampliaria o "feedback" positivo no hipotálamo e determinaria um pico de $\mathrm{LH}^{45}$. No presente estudo, sugere-se que a ação do eCG no crescimento final do folículo dominante, após a remoção do Norgestomet, possivelmente tenha ocorrido pela presença de um maior número de receptores de LH e FSH. Tal condição fisiológica favoreceria o incremento nas taxas de concepção em vacas com até 45 dias pós-parto. Dados semelhantes foram reportados, onde aplicação de eCG, após o tratamento com progestágenos em vacas de corte amamentando, com menos de 60 dias pós-parto, incrementou as TC. 14,27

No PPP2, a presença de uma menor inibição do eixo hipotálamo-hipofisário ${ }^{46} \mathrm{e}$ maiores concentrações de LH na corrente sangüínea favoreceu o crescimento folicular e a ovulação ${ }^{19,46,47}$. Tal fato, pode explicar a semelhança nas TC, que foram de $41,93 \% \mathrm{e}$ $44,44 \%$ para eCG e BE, respectivamente $(\mathrm{P}>0,05)$. Entretanto, a TC no grupo controle foi menor quando comparada aos grupos eCG e $\mathrm{BE}(\mathrm{P}<0,01)$. A dose de $\mathrm{BE}$ utilizada no presente estudo segue as recomendações da literatura. ${ }^{48}$ As vacas no PPP2, no momento da retirada do implante, o folículo dominante possui condições de desenvolver-se até o folículo pré-ovulatório, considerando haver LH suficiente ${ }^{49}$. Considerando a alta concentração de receptores de FSH nesta fase ${ }^{50}$, o eCG ativaria tais receptores e incrementaria as concentrações de AMPc intrafolicular ${ }^{24,44}$, 
evento que promoveria a ativação da enzimas intra-foliculares e aumentaria a produção de $\mathrm{E}_{2}{ }^{45}$.

No presente estudo conclui-se que a utilização do eCG associado ao Norgestomet promove um incremento nas taxas de concepção em vacas com até 45 dias pós-parto submetidas à IATF e com um período pósparto maior que 45 dias a utilização do eCG ou BE associado ao Norgestomet promoveu um incremento nas taxas de concepção em vacas submetidas à IATF.

\section{Effect of eCG or estradiol benzoate associated with norgestomet on the conception rate of beef cows submitted to FTAI in the postpartum}

\section{Abstract}

This study aimed to compare conception rates at the postpartum period (PPP) in beef cows which were administered either Equine Chorionic Gonadotrophin (eCG) or Estradiol Benzoate (EB) after Norgestomet and submitted to fixed-time artificial insemination (FTAI). The hypothesis was that the administration of eCG or EB enhances the conception rate. Lactating Nelore cows $(n=138)$ and Brangus ( $n=63)$, between 26 and 118 days of post partum period (PPP) were divided into three homogeneous groups, and subdivided in two blocks, inside each group one of them with a PPP d" 45 days (PPP1; $\mathrm{n}=107$ ) and another with a PPP $>45$ days (PPP2; $\mathrm{n}=94)$. All cows received an auricular implant containing $3 \mathrm{mg}$ Norgestomet $\left(\right.$ Crestar $\left.^{\circledR}\right)$, followed by the administration of $5 \mathrm{mg}$ Estradiol Valerate. The auricular implants were kept during 10 days. Cows received $1 \mathrm{~mL}$ saline solution ( $\mathrm{n}=68$, Control Group) or 500IU eCG (Folligon ${ }^{\circledR}$; $\mathrm{n}=67$; eCG Group) or 1mg Estradiol Benzoate (Index, $\mathrm{n}=66$; EB Group) 24 hours after the removal of the implant. FTAI was made 54 hours after the implant removal. The pregnancy diagnosis was carried through ultrasonography 30 days after FTAI. There was an interaction between treatments and PPP. In the PPP1, the conception rate was higher in the eCG Group than in the EB Group $47.22 \%$ vs. $15.38 \%$; Pd"0.01). In the PPP2, the conception rates of the eCG and EB Groups were higher than in the Control Group (41.93\%, 44.44\% vs. $22.22 \%$ : Pd"0.01). It was concluded that in cows up to 45 days of PPP, the eCG associated with Norgestomet enhances the conception rates.

\section{Referências}

1 BARUSELLI, P. S.; REIS, E. L.; MARQUES, M. O. Inseminação artificial em tempo fixo em bovinos de corte. In: SIMPÓSIO INTERNACIONAL DE REPRODUÇÃOANIMAL APLICADA, 1., 2004, Londrina. Anais... Londrina: [s.n.], 2004. p. 155-165.

2 MADUREIRA, E. H.; FERNANDES, R. H. R.; ROSSA, L. A. F.; PIMENTEL, J. R. V.; BRAGA, F. A.; PARDO, F. J. D. Anestro pós-parto em bovinos: a suplementação com óleos vegetais pode ser útil para encurtá-lo? In: ANAIS DO SIMPÓSIO INTERNACIONAL DE REPRODUÇÃO ANIMAL APLICADA, 2., 2006 , Londrina. Anais... Londrina: [s.n.], 2006. p. 63-70.

3 YAVAS, Y.; WALTON, J. S. Postpartum acyclicity in
Key words: Norgestomet. eCG.

Estradiol benzoate. Postpartum.

Cows. suckled beef cows: A review. Theriogenology, v. 54, n 1, p. 25-55, 2000b.

4 GASSER, C. L.; BEHLKE, E. J.; BURKE, C. R.; GRUM, D. E.; MUSSARD, M. L. improvement of pregnancy rate to fixed-time artificial insemination with progesterone treatment in anestrous pos-partum cows. Journal of Animal Science, v. 81, p. E45, 2003. Supplement. 2. Abstract.

5 LUCY, M. C.; BILLINGS, H. J.; BUTLER, W. R.; ENIS, L. R.; FIELDS, M. J.; KESLER, D. J.; KINDER, J. E.; MATTOS, R. C.; SHORT, R. E.; THATCHER, W. W.; WETTMAN, R. P.; YELICH, J. V.; HAFS, H. D. Efficacy of an intravaginal progesterone insert and injection of PGF2a for synchronizing estrus and shortening the interval to pregnancy in postpartum beef cows, peribubertal beef heifers and dairy heifers. Journal of 
Animal Science, v. 79, n. 4, p. 982-995, 2001.

6 HESS, B. W.; LAKE, S. L.; SCHOLLJEGERDES, E. J.; WESTON, T. R.; NAYIGIHUGU, V.; MOLLE, J. D. C.; MOSS, G. E. Nutritional controls of beef cow reproduction. Journal of Animal Science, v. 83, n. 13, p. E90-106, 2005. Supplement, 1.

7 WETTMANN, R. P.; LENTS, C. A.; CICCIOLI, N. H.; WHITE, F. J.; RUBIO, I. Nutritional and suckling mediated anovulation in beef cows. Journal of Animal Science, v. 81, n. 14, p. E48-59, 2003. Supplement, 2.

8 MADUREIRA, E. H.; PIMENTEL, J. R. V.; ALMEIDA, A. B.; ROSSA, L. A. F. Sincronização com progestágenos. In: ANAIS DO SIMPÓSIO INTERNACIONAL DE REPRODUÇÃO ANIMAL APLICADA, 1., 2004, Londrina. Anais... Londrina: [s.n.], 2004. p. 117-128.

9 NETT, T. M. Function of the hypothalamichypophysial axis during the postpartum period in ewes and cows. Journal Reproduction in Domestic Ruminants, v. 34, p. 210-13, 1987.

10 MIHM, M. Delayed resumption of ciclicity in postpartum dairy and beef cattle. Reproduction Domestic Animal, v. 34, p. 277-284, 1999.

11 WILLIAMS, G. L. Suckling as a regulator of postpartum rebreeding in cattle: a review. Journal of Animal Science, v. 68, n. 3, p. 831-852, 1990

12 WILLIAMS, G. L.; TALAVERA, B. J.; PETERSEN, B. J.; KIRSCH, J. D.; TILTON, J. E. Coincident secretion of follicle-stimulating hormone and luteinizing hormone in early postpartum beef cows: effects of suckling and low-level increases of systemic progesterone. Biology of Reproduction, v. 29, n. 2, p. 362-373, 1983.

13 SCHALLENBERGER, E. Gonadotrophins and ovarian steroids in cattle. Pulsatile changes of gonadotrophin concentrations in the jugular vein postpartum. Acta Endocrinology, v. 109, p. 37-43, 1985.

14 ROCHE, J. F.; CROWE, M. A.; BOLAND, M. P. Pospartum anoestrus in dairy and beef cows. Animal Reproduction Science, v. 38, p. 371-378, 1992.

15 JOLY, P. D.; MCDOUGALL, S.; FITZPATRICK, L. A.; MACMILLAN, K. L.; ENTWISTLE, K. W. Physiological effects of under nutrition on postpartum anoestrum in cows. Journal of Reproduction and Fertility, v. 39, p. 477-492, 1995.

16 GARCIA-WINDER, M.; LEWIS, P. E.; DEAVER, D. R.; SMITH, V. G.; LEWIS, G. S.; INSKEEP, E. K. Endocrine profiles associated with life span of induced corpora lutea in postpartum beef cows. Journal of Animal Science, v. 62, p. 1353-1362, 1986.

17 KESLER, D. J.; GARVERICK, H. A.; YOUNGQUIST, R. S.; ELMORE, R. G.; BIERSCWAL, C. J. Effects of days postpartum and endogenous reproductive hormones on $\mathrm{GnRH}$-induced LH release dairy cows. Journal of Animal Science, v. 46, p. 797-803, 1977.

18 STAGG, K.; DISKIN, M. G.; SREENAN, J. M.;
ROCHE, J. F. Folicular development in long-term anoestrous suckler beef cows fed two levels of energy postpartum. Animal Reproduction Science, v. 38, p. 49-61, 1995.

19 GARCIA-WINDER, M.; LEWIS, P. E.; TOWNSEND, E. C.; INSKEEP, E. K. Effects of Norgestomet on follicular development in postpartum beef cows. Journal of Animal Science, v. 64, p. 104-112, 1987

20 GARVERICK, H. A.; PARFET, J. R.; LEE, C. N.; COPELIN, J. P.; YOUNGQUIST, R. S.; SMITH, M. F. Relationship of pre and post-ovulatory gonadotropin concentrations to subnormal luteal function in postpartum beef cattle. Journal of Animal Science, v. 66, p. 104-111, 1988.

21 YAVAS, Y.; WALTON, J. S. Induction of ovulation in postpartum suckled beef cows: A review. Theriogenology, v. 54, n. 1, p. 1-25, 2000a.

22 BEAL, W. E.; GOOD, G. A.; PETERSON, L. A. Estrus synchronization and pregnancy rates in cyclic and noncyclic beef cows and heifers treated with SyncroMate-B or norgestomet and alfaprostol. Theriogenology, v. 22, n. 1 , p. 59-66, 1984

23 YAVAS, Y.; JOHNSON, W. H.; WALTON, J. S. Modification of follicular dynamics by exogenous FSH and progesterone, and the induction of ovulation using hCG in postpartum beef cows. Theriogenology, v. 52, n. 6, p. 949-963, 1999

24 MURPHY, B. D.; MARTINUK, S. D. Equine chorionic gonadotrophin. Endocrine Rewiews, v. 12, p. 27-44, 1991.

25 CAVALIERI, J.; RUBIO, I.; KINDER, J. E.; ENTWISTLE, K. W.; FITZPATRICK, L. A. Synchronization of estrus and ovulation and associated endocrine changes in Bos indicus cows. Theriogenology, v. 47, n. 4, p. 801-814, 1997.

26 HANLON, D. W.; WILLIANSON, N. B.; WICHTEL, J. J.; STEFFERT, I. J.; CRAIGIE, A. L.; PFEIFFER, D. U. The effect of Estradiol benzoate administration on oestrus response and synchronization pregnancy rate in dairy heifers after treatment with exogenous progesterone. Theriogenology, v. 45, n. 4, p. 775-785, 1996.

27 BO, G. A.; CUTAIA, I.; BARUSELLI, P. S. Programas de inseminacion artificial y transferencia de embriones a tiempo fijo. In: SIMPÓSIO INTERNACIONAL DE REPRODUÇÃOANIMAL APLICADA, 1., 2004 , Londrina. Anais... Londrina: [s.n.], 2004. p. 56-81.

28 RICHARDS, M. W.; SPITZER, J. C.; WARNER, M. B. Effect of varying levels of postpartum nutrition and body condition at calving on subsequent reproductive performance in beef cattle. Journal of Animal Science, v. 62 , n. 2 , p. $300-306,1986$.

29 STATISTICAL ANALISYS SYSTEM. SAS user's guide: statistics. 5ed. Cary: SAS Institute, 1985.

30 SINGH, U.; KHURANA, N. K.; INDERJEET. Plasma progesterone profiles and fertility status of anestrus zebu 
cattle treated with Norgestomet-estradiol-eCG regimen. Theriogenology, v. 50, n. 8, p. 1191-1199, 1998.

31 SMITH, M. F.; BURRELL, W. C.; SHIPP, L. D.; SPROTT, L. R.; SONGSTER, W. N.; WILTBANK, J. N. Hormone treatments and use calf removal in postpartum beef cows. Journal of Animal Science, v. 48, p. 12851294, 1979.

32 MULVEHILL, P.; SREENAN, J. M. Improvement of fertility in postpartum beef cows by treatment with PMSG and prosgestagen. Journal of Reproduction and Fertility, v. 50, p. 323-325, 1977.

$33 \mathrm{XU}$, Z. Z.; GARVERICK, H. A.; SMITH, G.; SMITH, M. F.; HAMILTON, S. A.; YOUNGQUIST, R. S. Expression of $\mathrm{FSH}$ and $\mathrm{LH}$ receptor messenger ribonucleic acids in bovine follicles during the first follicular wave. Biology of Reproduction, v. 53, p. 951-957, 1995.

34 WEBB, R.; CAMPELLI, B. K.; GARVERICK, H. A.; GONG, J. G.; GUTIERREZ, C. G.; ARMSTRONG, D. G. Molecular mechanisms regulating follicular recruitment and selection. Journal of Reproducyion and Fertility, v. 45, p. 33-48, 1999. Supplement.

35 INSKEEP, E. K.; BRADEN, T. D.; LEWIS, P. E.; GARCIA-WINDER, M.; NISWENDER, G. D. Receptors for $\mathrm{LH}$ and $\mathrm{FSH}$ in large follicle in postpartum beef cows. Biology of Reproduction, v. 38, p. 587-591, 1988.

36 KASTELIC, J. P.; OLSON, W. O.; MARTINEZ, M. A.; MAPLETOFT, R. J.; MACHADO, R. Sincronização do estro em bovinos Hereford-Angus com Crestar. Revista Brasileira de Reprodução Animal, v. 21, p. 101-103, 1997.

37 HUMBLOT, P.; GRIMARD, B.; MIALOT, J. P. Sorces of variation of post-partum cyclicity, ovulation and pregnancy rates in suckled beef cows treated with progestagen and PMSG. In: ANNUAL MEETING OF THE SOCIETY FOR THERIOGENOLOGY, 1996, Kansas City. Proceedings... 1996. p. 36-45.

38 SCENA, C. Uso de implantes progestágenos subcutaneous para inducer y sincronizar cellos en rodeos de cría. IN: CUERTAS JORNADAS NACIONALES CABIA Y PRIMERAS DEL MERCOSUR, 1998, Buenos Aires, Argentina. 1998. p. 59-68.

39 BÓ, G. A.; CUTAIA, L.; TRIBULO, R. Tratamientos hormonales para inseminación artificial a tiempo fijo em bovinos para carne: algunas experiências realizadas em Argentina. Primeira Parte. Taurus, v. 14, p. 10-21, 2002b.

40 BÓ, G. A.; CUTAIA, L.; TRIBULO, R. Tratamientos hormonales para inseminación artificial a tiempo fijo em bovinos para carne: algunas experiências realizadas em Argentina. Segunda Parte. Taurus, v. 15, p. 17-32, 2002c
41 RHIND, S. M.; BRAMLEY, T. A.; WRIGHT, L. A.; MCMILLEN, S. R. FSH and LH receptor concentration in large ovarian follicle of beef cows in high and low levels of body condition at nine weeks pos partum. Reproduction Fertility and Development, v. 4, p. 515522, 1992.

42 CROWE, M. A.; KELLY, P.; DRIANCOURT, M. A.; BOLAND, M. P.; ROCHE, J. F. Effects of FSH with and without $\mathrm{LH}$ on serum hormone concentration, follicle growth and intrafollicular estradiol and aromatase activity in GnRH - immunized heifers. Biology of Reproduction, v. 64, p. 368-374, 2001.

43 GONG, J. G.; CAMPBELL, B. K.; BRAMLEY, T. A.; GUTIERREZ, C. G.; PETERS, A. R.; WEBB, R. Suppression in the secretion of follicle-stimulating hormone and luteinizing hormone, and ovarian follicle development in heifers continuosly infused with a gonadotropin-releasing hormone agonist. Biology of Reproduction, v. 55, p. 68-74, 1996.

44 FORTUNE, J. E.; QUIRK, S. M. Regulation of esteroidogenesis in bovine preovulatory follicles. Journal of Animal Science, v. 66, p. 1-8, 1988. Supplement, 2.

45 VOOS, A. K.; FORTUNE, J. E. Levels of messenger ribonucleic acid for cytocrome P450 and $17 \mathrm{~b}$ hydroxylase and $\mathrm{P} 450$ aromatase in bovine preovulatory follicles after the LH surge. Endocrinology, v. 132, p. 2239-2245, 1993.

46 NETT, T. M.; CERMARK, D.; BRADEN, T.; MANNS, J.; NISWENDER, G. Pituitary receptor for GnRH and estradiol and pituitary content of gonadotophins in beef cows II. Domestic Animal Endocrinology, v. 5, p. 8189, 1988.

47 CAVALIERI, J.; FITZPATRICK, L. A. Oestrus detection techniques and insemination strategies in Bos indicus heifers synchronized with Norgestomet-estradiol. Australian Veterinary Journal, v. 72, p. 177-182, 1995.

48 MACMILLAN, K. L.; PETERSON, A. J. A new intravaginal progesterone releasing devices for cattle (CIDR-B) for oestrous synchronization, increasing pregnancy rates and the treatment of post-partum anoestrus. Animal Reproduction Science, v. 33, n. 1, p. 1-25, 1993.

49 GINTHER, O. J.; BERGFELT, D. R.; KULICK, L. J.; KOT, K. Selection of dominante folliclein cattle: establishment of follicle deviation in less than 8 hours through depression of $\mathrm{FSH}$ concentration. Theriogenology, v. 52, p. 1079-1093, 1999.

50 WEBB, R.; CAMPBELLI, B. K.; GARVERICK, H. A.; GONG, J. G.; GUTIERREZ, C. G.; ARMSTRONG, D. G. Molecular mechanisms regulating follicular recruitment and selection. Journal of Reproduction and Fertility, v. 54, p. 33-48, 1999. Supplement. 